\title{
Combination therapy of molecular hydrogen and hyperoxia improves survival rate and organ damage in a zymosan-induced generalized inflammation model
}

\author{
YUNCHUAN HONG ${ }^{1 *}$, LI SUN $^{2 *}$, RUIQIANG SUN ${ }^{3 *}$, HONGGUANG CHEN $^{4}$, YONGHAO YU ${ }^{4}$ and KELIANG XIE ${ }^{4,5}$ \\ ${ }^{1}$ Department of Respiratory Medicine, General Hospital of Tianjin Medical University, Tianjin 300052; \\ ${ }^{2}$ Department of Anesthesiology, General Hospital of Beijing Military Command, Beijing 100700; \\ ${ }^{3}$ Department of Anesthesiology, Tianjin Eye Hospital, Tianjin Key Lab of Ophthalmology and Visual Science, Tianjin 300020; \\ ${ }^{4}$ Department of Anesthesiology, Tianjin Institute of Anesthesiology, General Hospital of Tianjin Medical University, \\ Tianjin 300052; ${ }^{5}$ Department of Anesthesiology, Clinical Medical School of Yangzhou University, \\ Yangzhou, Jiangsu 225001, P.R. China
}

Received December 20, 2014; Accepted February 11, 2016

DOI: $10.3892 /$ etm.2016.3231

\begin{abstract}
Multiple organ dysfunction syndrome (MODS) is a leading cause of mortality in critically ill patients. Hyperoxia treatment may be beneficial to critically ill patients. However, the clinical use of hyperoxia is hindered as it may exacerbate organ injury by increasing reactive oxygen species (ROS). Hydrogen gas $\left(\mathrm{H}_{2}\right)$ exerts a therapeutic antioxidative effect by selectively reducing ROS. Combination therapy of $\mathrm{H}_{2}$ and hyperoxia has previously been shown to significantly improve survival rate and organ damage extent in mice with polymicrobial sepsis. The aim of the present study was to investigate whether combination therapy with $\mathrm{H}_{2}$ and hyperoxia could improve survival rate and organ damage in a zymosan (ZY)-induced generalized inflammation model. The results showed that the inhalation of $\mathrm{H}_{2}(2 \%)$ or hyperoxia (98\%) alone improved the 14-day survival rate of ZY-challenged mice from
\end{abstract}

Correspondence to: Dr Keliang Xie, Department of Anesthesiology, Tianjin Institute of Anesthesiology, General Hospital of Tianjin Medical University, 154 Anshan Road, Tianjin 300052, P.R. China

E-mail: xiekeliang2009@hotmail.com

*Contributed equally

Abbreviations: 8-iso-PGF2 $\alpha, 8$-iso-prostaglandin F2 $\alpha$; ALT, alanine aminotransferase; AST, aspartate aminotransferase; BUN, blood urea nitrogen; CAT, catalase; CLP, cecal ligation and puncture; $\mathrm{Cr}$, creatinine; GSH-Px, glutathione peroxidase; $\mathrm{H}_{2}$, hydrogen; $\mathrm{H}_{2} \mathrm{O}_{2}$, hydrogen peroxide; HMGB1, high-mobility group box 1; ICU, intensive care units; MODS, multiple organ dysfunction syndrome; NS, normal saline; $\bullet \mathrm{OH}$, hydroxyl radicals; ROS, reactive oxygen species; SOD, superoxide dismutase; ZY, zymosan

Key words: multiple organ dysfunction syndrome/failure, reactive oxygen species, inflammatory cytokines, hydrogen gas, hyperoxia
20 to 70 or $60 \%$, respectively. However, combination therapy with $\mathrm{H}_{2}$ and hyperoxia could increase the 14-day survival rate of ZY-challenged mice to $100 \%$. Furthermore, ZY-challenged mice showed significant multiple organ damage characterized by increased serum levels of aspartate transaminase, alanine transaminase, blood urea nitrogen and creatinine, as well as lung, liver and kidney histopathological scores at $24 \mathrm{~h}$ after ZY injection. These symptoms where attenuated by $\mathrm{H}_{2}$ or hyperoxia alone; however, combination therapy with $\mathrm{H}_{2}$ and hyperoxia had a more marked beneficial effect against lung, liver and kidney damage in ZY-challenged mice. In addition, the beneficial effects of this combination therapy on ZY-induced organ damage were associated with decreased serum levels of the oxidative product 8-iso-prostaglandin F2 $\alpha$, increased activity of superoxide dismutase and reduced levels of the proinflammatory cytokines high-mobility group box 1 and tumor necrosis factor- $\alpha$. In conclusion, combination therapy with $\mathrm{H}_{2}$ and hyperoxia provides enhanced therapeutic efficacy against multiple organ damage in a ZY-induced generalized inflammation model, suggesting the potential applicability of $\mathrm{H}_{2}$ and hyperoxia in the therapy of conditions associated with inflammation-related MODS.

\section{Introduction}

Multiple organ dysfunction syndrome (MODS) is the leading cause of mortality in critically ill patients (1). MODS is defined as the progressive deterioration of organ function, which often occurs in patients with severe sepsis, septic shock, hemorrhagic shock, multiple trauma, severe burns or pancreatitis (2). The mechanisms underlying the pathology of MODS are not fully understood (3), therefore it is difficult to develop an effective therapeutic measure for patients with MODS.

Oxygen inhalation is frequently administered to critically ill patients. Early goal-directed therapy for MODS aims to balance tissue oxygen delivery and oxygen demand. Previous studies have suggested that hyperoxia may have a beneficial effect against sepsis and sepsis-associated multiple organ 
damage (4-8). However, the clinical use of hyperoxia is limited in critically ill patients due to concerns that it may exacerbate organ damage by increasing free radical formation. Oxidative stress is crucially involved in the pathogenesis of MODS, and overproduction of reactive oxygen species (ROS) may exacerbate organ damage $(9,10)$. It has been suggested that a low concentration of molecular hydrogen $\left(\mathrm{H}_{2}\right)$ exerts a therapeutic antioxidative effect by selectively reducing hydroxyl $\left({ }^{\circ} \mathrm{OH}\right)$ radicals, the most cytotoxic ROS, and peroxynitrite $\left(\mathrm{ONOO}^{-}\right)$, and effectively protects against numerous diseases (11-15). Furthermore, our previous study showed that combination therapy with $\mathrm{H}_{2}$ and hyperoxia can significantly alleviate organ injury and improve survival rate of mice with polymicrobial sepsis via reducing oxidative stress and inflammation (16).

Therefore, these findings strongly indicate that combination therapy with $\mathrm{H}_{2}$ and hyperoxia may afford a more potent therapeutic strategy for sepsis. The zymosan (ZY)-induced generalized inflammation model has been widely used by other research groups (17), as well as by our group (8), because $\mathrm{ZY}$, a substance derived from the cell wall of the yeast Saccharomyces cerevisiae, can produce systemic inflammation by inducing a wide range of inflammatory mediators (18). This model has also been used in previous experimental studies investigating MODS (18). Therefore, the aim of the present study was to investigate whether combination therapy with $\mathrm{H}_{2}$ and hyperoxia could produce enhanced efficacy in a murine model of ZY-induced generalized inflammation.

\section{Materials and methods}

Animals. Male Imprinting Control Region (ICR) mice (specific pathogen-free; age, 6-8 weeks; weight, 20-25 g) provided by the Laboratory Animal Center of the Academy of Military Science of the Chinese People's Liberation Army (Beijing, China) were used in all experiments. Animals were housed at $20-22^{\circ} \mathrm{C}$ with a 12 -h light/dark cycle. Animals were fed standard chow and water ad libitum. All experimental protocols were approved by the Institutional Animal Care and Use Committee of Tianjin Medical University (Tianjin, China), and were performed in accordance with the National Institutes of Health guidelines for the use of experimental animals (19).

ZY-induced generalized inflammation model. ZY (Sigma-Aldrich, St. Louis, MO, USA) was dissolved in an isotonic sodium chloride solution (normal saline [NS]) to a final concentration of $25 \mathrm{mg} / \mathrm{ml}$ and was sterilized at $100^{\circ} \mathrm{C}$ for $80 \mathrm{~min}$. All suspensions were freshly produced prior to use. Generalized inflammation was induced by an aseptic intraperitoneal injection of ZY $(1 \mathrm{~g} / \mathrm{kg})(8,18)$. The same volume of NS was injected via the same route as a control.

Molecular hydrogen and/or hyperoxia treatment. The animals were put in a sealed plexiglass chamber with inflow and outflow outlets $(20,21) . \mathrm{H}_{2}, \mathrm{O}_{2}$ or $\mathrm{N}_{2}$ was supplied via a gas flowmeter, (Yukata Engineering Corp., Tokyo, Japan) and delivered into the chamber through a tube at a rate of $4 \mathrm{l} / \mathrm{min}$. The concentrations of $\mathrm{O}_{2}$ and $\mathrm{H}_{2}$ in the chamber were continuously monitored using a gas analyzer (LB-2, Model 40 M; Beckman Coulter, Inc., Fullerton, CA, USA) and a commercially available hydrogen detector (HY-ALERTA ${ }^{\mathrm{TM}}$ 500, H2scan Corporation,
Valencia, CA, USA), respectively. $\mathrm{CO}_{2}$ was removed from the chamber gases using baralyme (Allied Healthcare Products, Inc., St. Louis, MO, USA). The mixed gases were maintained at the following levels: $2 \% \mathrm{H}_{2}, 21 \% \mathrm{O}_{2}$ and $77 \% \mathrm{~N}_{2} ; 0 \% \mathrm{H}_{2}$, $98 \% \mathrm{O}_{2}$ and $2 \% \mathrm{~N}_{2}$; and $2 \% \mathrm{H}_{2}, 98 \% \mathrm{O}_{2}$ and $0 \% \mathrm{~N}_{2}$ during the treatment. The animals without $\mathrm{H}_{2}$ or hyperoxia treatment were exposed to room air in the chamber.

\section{Experimental design}

Experiment one: Effects of $\mathrm{H}_{2}$ and/or hyperoxia treatment on the survival rate in $Z Y$-challenged mice. A total of 180 animals were randomly allocated into six groups $(n=30$ per group): Normal saline (NS), $\mathrm{NS}+\mathrm{H}_{2}+\mathrm{O}_{2} ; \mathrm{ZY} ; \mathrm{ZY}+\mathrm{H}_{2}$; $\mathrm{ZY}+\mathrm{O}_{2}$; and $\mathrm{ZY}+\mathrm{H}_{2}+\mathrm{O}_{2}$ groups. The treatment concentrations of hydrogen and hyperoxia were determined based on our previous studies and preliminary observations $(8,21)$. The animals in the $\mathrm{ZY}+\mathrm{O}_{2}$ and $\mathrm{ZY}+\mathrm{H}_{2}$ groups were exposed to $98 \% \mathrm{O}_{2}$ or $2 \% \mathrm{H}_{2}$ for $3 \mathrm{~h}$ starting at 1 and $6 \mathrm{~h}$ after $\mathrm{ZY}$ injection, respectively. The animals in the $\mathrm{NS}+\mathrm{H}_{2}+\mathrm{O}_{2}$ and $\mathrm{ZY}+\mathrm{H}_{2}+\mathrm{O}_{2}$ groups were exposed to $98 \% \mathrm{O}_{2}$ and $2 \% \mathrm{H}_{2}$ at the same time points. As a control, the animals from the NS and ZY groups were given room air treatment at the same time points. The survival rate was observed on days $1,2,3,4,5,6$, 7 and 14 after NS or ZY injection. In addition, arterial blood gas evaluation was conducted at $1.5 \mathrm{~h}$ after the onset of $\mathrm{H}_{2}$ inhalation (2.5 h after NS or ZY injection) in all groups.

Experiment two: Effects of $\mathrm{H}_{2}$ and/or hyperoxia treatment on organ injury in ZY-challenged mice. Additional 36 animals were used in this experiment and were assigned to six groups ( $n=6$ per group). The grouping method and experimental protocols were the same as Experiment One. At $24 \mathrm{~h}$ after NS or ZY injection, all the animals were sacrificed with sodium pentobarbital (50 mg/kg, intraperitoneally), and the blood samples and organs were collected for detecting serum biochemical parameters and organ histopathology.

In addition, at $24 \mathrm{~h}$ after NS or ZY injection, the serum levels of the proinflammatory cytokines high-mobility group box 1 (HMGB1) and tumor necrosis factor- $\alpha$ (TNF- $\alpha$ ), the antioxidative enzyme superoxide dismutase (SOD) and the oxidative product 8 -iso-prostaglandin F2 $\alpha$ (8-iso-PGF $2 \alpha$ ) were evaluated.

Arterial blood gas analysis. The arterial blood gas analysis (for $\mathrm{pH}, \mathrm{PaO}_{2}$ and $\mathrm{PaCO}_{2}$ ) was conducted using a GEM Premier 3000 gas analyzer (Instrumentation Laboratory Spa, Milan, Italy).

Serum biochemical parameters assay. The serum was separated from whole blood samples by centrifugation at $1,100 \mathrm{x} g$ for $10 \mathrm{~min}$, aliquoted and stored at $-80^{\circ} \mathrm{C}$ until assayed $(8,20-22)$. The samples were evaluated using a biochemistry autoanalyzer (7150; Hitachi, Ltd., Tokyo, Japan) to measure serum levels of alanine aminotransferase (ALT, IU/l), aspartate aminotransferase (AST, IU/l), blood urea nitrogen (BUN, mmol/l) and creatinine $(\mathrm{Cr}, \mu \mathrm{mol} / \mathrm{l})$.

Organ histological examination. The lung, liver and kidneys were removed immediately, fixed in $4 \%$ paraformaldehyde (Sigma-Aldrich), embedded in paraffin, and sectioned at 4-6 $\mu \mathrm{m}$. After deparaffinization and rehydration, the sections 


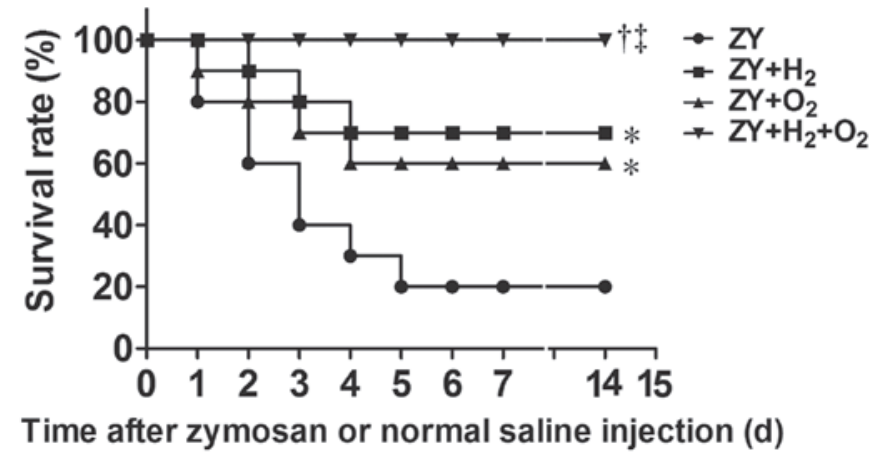

Figure 1. Effects of $\mathrm{H}_{2}$ and/or hyperoxia on the survival rate of zymosan-challenged mice. The mice were treated with or without $2 \% \mathrm{H}_{2}$ and/or $98 \% \mathrm{O}_{2}$ inhalation for $3 \mathrm{~h}$ starting at 1 and $6 \mathrm{~h}$ after normal saline or zymosan injection, respectively. Values are expressed as survival percentage ( $\mathrm{n}=30$ per group). ${ }^{*} \mathrm{P}<0.05$ vs. $\mathrm{ZY}$ group; ${ }^{\dagger} \mathrm{P}<0.05$ vs. $\mathrm{ZY}+\mathrm{H}_{2}$ group; ${ }^{\ddagger} \mathrm{P}<0.05$ vs. $\mathrm{ZY}+\mathrm{O}_{2}$ group. $\mathrm{ZY}$, zymosan; d, days.

were stained with hematoxylin and eosin (Sigma-Aldrich). Based on the scoring standard in our previous studies $(8,22)$, the histological slides were blindly examined and scored by two experienced pathologists.

Detection of SOD activity. The activities of SOD were measured using commercial kits purchased from Cayman Chemical Company (Ann Arbor, MI, USA). Total SOD activity was assayed according to the manufacturer's instructions and the protocol described in our previous studies (8,20-22). All spectrophotometric readings were performed using a spectrophotometer (DU-640B; Beckman Coulter, Inc.). All assays were conducted in triplicates.

Detection of 8-iso-PGF2 $\alpha$. Measurement of 8 -iso-PGF2 $\alpha$, a free radical-catalyzed product of arachidonic acid, can offer a reliable approach for quantitative measurement of oxidative stress status in vivo (23). The levels of serum 8-iso-PGF2 $\alpha$ were detected using specific enzyme-linked immunosorbent assay (ELISA) kits (cat. no. 516351; Cayman Chemical Company) and a Spectramax M5 microplate reader (Molecular Devices, LLC, Sunnyvale, CA, USA) (8,20-22). All standards and samples were run in duplicate.

Detection of inflammatory cytokines. The levels of serum TNF- $\alpha$ (R\&D Systems, Inc., Minneapolis, MI, USA) and HMGB1 (IBL International GmbH, Hamburg, Germany) were detected using specific ELISA kits and the Molecular Devices microplate reader (8,20-22). All standards and samples were run in duplicate.

Statistical analysis. Survival rates are expressed as percentages and measurement data are expressed as the mean \pm standard error of the mean. The analysis of survival rates were tested using Fisher's exact probability method. The inter-group differences of the rest data were tested by one-way analysis of variance followed by Least Significant Difference t-test for multiple comparisons. Statistical analyses were performed using SPSS software, version 16.0 (SPSS, Inc., Chicago, IL, USA). $\mathrm{P}<0.05$ was considered to indicate a statistically significant difference.

\section{Results}

Differences in arterial blood gas during treatment. In the present study, the effects of $\mathrm{H}_{2}$ inhalation on arterial $\mathrm{pH}$, $\mathrm{PaO}_{2}$ and $\mathrm{PaCO}_{2}$ were evaluated in all groups at $1.5 \mathrm{~h}$ after the onset of $\mathrm{H}_{2}$ and/or $\mathrm{O}_{2}$ inhalation $(2.5 \mathrm{~h}$ after $\mathrm{NS}$ or $\mathrm{ZY}$ injection). The levels of $\mathrm{P}_{\mathrm{a}} \mathrm{O}_{2}$ in the $\mathrm{ZY}+\mathrm{O}_{2}, \mathrm{ZY}+\mathrm{H}_{2}+\mathrm{O}_{2}$ and $\mathrm{NS}+\mathrm{H}_{2}+\mathrm{O}_{2}$ groups were 405.1 \pm 19.2 , 410.3 \pm 18.7 and $408.4 \pm 21.5 \mathrm{mmHg}$, respectively. The $\mathrm{P}_{\mathrm{a}} \mathrm{O}_{2}$ levels in the NS, $\mathrm{ZY}$ and $\mathrm{ZY}+\mathrm{H}_{2}$ groups were $97.18 \pm 8.29$, 96.72 \pm 10.31 and $95.46 \pm 8.46 \mathrm{mmHg}$, respectively. There were no differences in the levels of arterial $\mathrm{pH}$ and $\mathrm{P}_{\mathrm{a}} \mathrm{CO}_{2}$ among all groups (data not shown). The results demonstrate that hyperoxia exposure can significantly increase the $\mathrm{P}_{\mathrm{a}} \mathrm{O}_{2}$ level, and $2 \% \mathrm{H}_{2}$ has no marked effects on the $\mathrm{P}_{\mathrm{a}} \mathrm{O}_{2}$ level in $\mathrm{ZY}$-challenged mice during the treatment.

Combination therapy with $\mathrm{H}_{2}$ and hyperoxia improves the survival rate in $Z Y$-challenged mice. The 14-day survival rate of ZY-challenged mice was $20 \%$ ( $\mathrm{P}<0.05$ vs. NS group; Fig. 1). The results showed that either $98 \% \mathrm{O}_{2}$ or $2 \% \mathrm{H}_{2}$ exposure for $3 \mathrm{~h}$ starting at 1 and $6 \mathrm{~h}$ after $\mathrm{ZY}$ injection, respectively, improved the 14-day survival rate of ZY-challenged mice to 60 or $70 \%$ ( $\mathrm{P}<0.05$ vs. ZY group; Fig. 1). Furthermore, combination therapy with $2 \% \mathrm{H}_{2}$ and $98 \% \mathrm{O}_{2}$ increased the 14-day survival rate of $\mathrm{ZY}$-challenged mice to $100 \%$ ( $\mathrm{P}<0.05$ vs. ZY group; Fig. 1). In addition, all mice in the $\mathrm{NS}$ and $\mathrm{NS}+\mathrm{H}_{2}+\mathrm{O}_{2}$ groups survived during the experiment. These results suggest that combination therapy with $\mathrm{H}_{2}$ and hyperoxia can improve the survival rate of ZY-challenged mice in a synergistic manner.

Combination therapy with $\mathrm{H}_{2}$ and hyperoxia improves organ histopathological scores in ZY-challenged mice. At $24 \mathrm{~h}$ after ZY or NS injection, the animals in all groups were killed for histopathological analysis. According to the scoring standard in our previous studies $(8,22)$, the histopathological changes in lung, liver and kidney were scored using a scale of 1 to 4 . As shown in the Fig. 2, the histopathological scores for lung, liver and kidney in the ZY group were 3-3.5, significantly increased compared with the NS group $(\mathrm{P}<0.05)$. These abnormal changes in $\mathrm{ZY}$-challenged mice were attenuated by either $98 \%$ $\mathrm{O}_{2}$ or $2 \% \mathrm{H}_{2}$ treatment alone ( $\mathrm{P}<0.05$ vs. ZY group; Fig. 2). Furthermore, these abnormal changes in ZY-challenged mice were more markedly ameliorated by combination therapy with $98 \% \mathrm{O}_{2}$ and $2 \% \mathrm{H}_{2}$ compared with either treatment alone (Fig. 2). These data indicate that combination therapy with $\mathrm{H}_{2}$ and hyperoxia has an enhanced efficacy against multiple organ damage in ZY-challenged mice.

Combination therapy with $\mathrm{H}_{2}$ and hyperoxia improves serum biochemical parameters in $Z Y$-challenged mice. As shown in Fig. 3, the ZY-challenged mice appeared significantly impaired liver and kidney function at $24 \mathrm{~h}$, which was assessed by serum biochemical parameters for liver and kidney function (ALT, AST, Cr and BUN). The ZY-challenged mice showed a significant increase in the levels of serum ALT, AST, Cr and BUN ( $\mathrm{P}<0.05$ vs. NS group), which were significantly attenuated by $2 \% \mathrm{H}_{2}$ or $98 \% \mathrm{O}_{2}$ treatment alone (Fig. 3). Furthermore, these abnormal changes of biochemical parameters in ZY-challenged mice were more notably ameliorated by combination therapy 

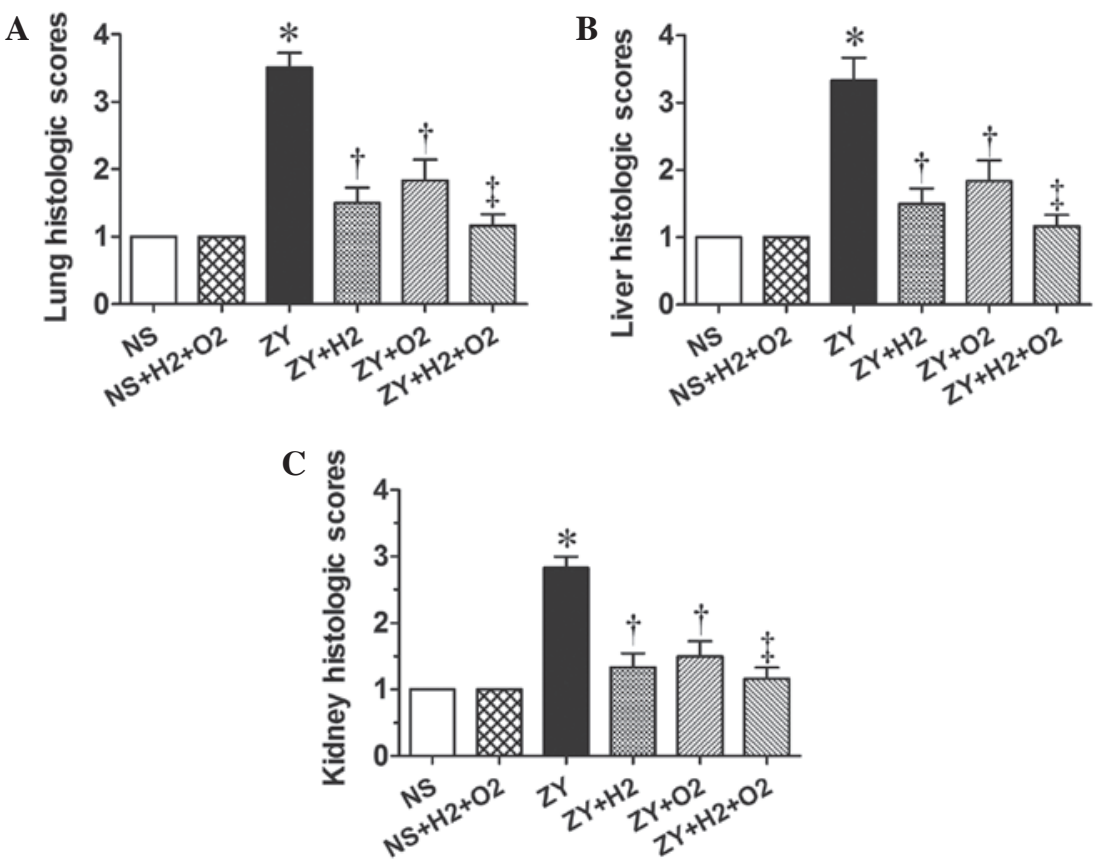

Figure 2. Effects of $\mathrm{H}_{2}$ and/or hyperoxia on the organ histopathological scores in zymosan-challenged mice. Histological scores in the mouse (A) lungs, (B) liver and (C) kidneys. Mice were treated with or without $2 \% \mathrm{H}_{2}$ and/or $98 \% \mathrm{O}_{2}$ inhalation for $3 \mathrm{~h}$ starting at 1 and $6 \mathrm{~h}$ after NS or zymosan injection, respectively. At $24 \mathrm{~h}$ after NS or zymosan injection, all animals were anesthetized, and the organ samples were collected for measuring the histopathological scores. Values are expressed as the mean \pm standard error of the mean ( $\mathrm{n}=6$ per group). ${ }^{\mathrm{P}} \mathrm{P}<0.05$ vs. NS group; ${ }^{\mathrm{P}}<0.05$ vs. $\mathrm{ZY}$ group; ${ }^{{ }^{*}} \mathrm{P}<0.05$ vs. $\mathrm{ZY}+\mathrm{O}_{2}$ group. NS, normal saline; ZY, zymosan.

A
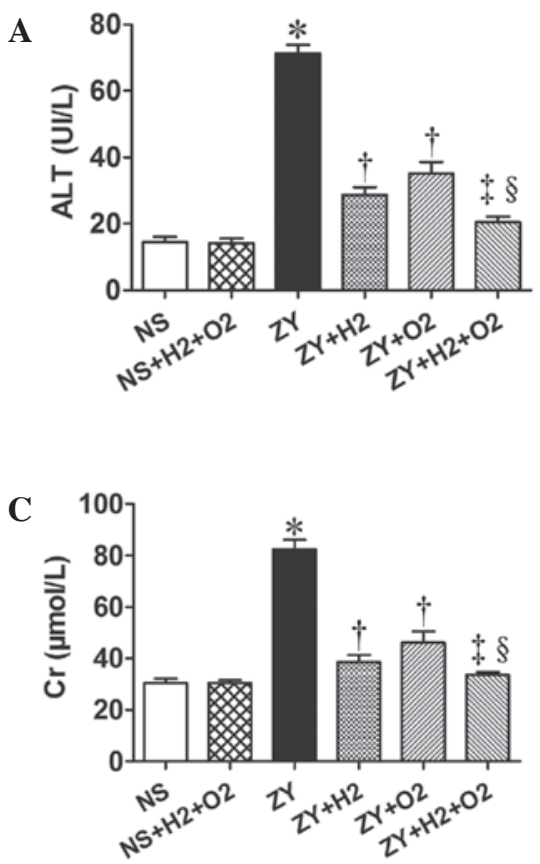

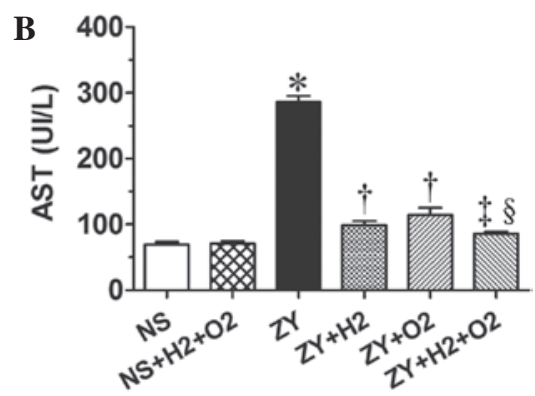

D

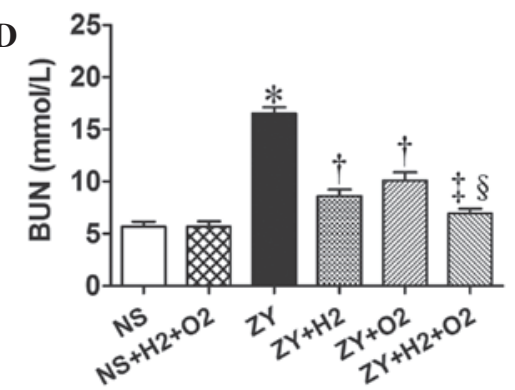

Figure 3. Effects of $\mathrm{H}_{2}$ and/or hyperoxia on the serum biochemical parameters in zymosan-challenged mice. Serum levels of (A) ALT, (B) AST, (C) Cr and (D) BUN. Mice were treated with or without $2 \% \mathrm{H}_{2}$ and/or $98 \% \mathrm{O}_{2}$ inhalation for $3 \mathrm{~h}$ starting at 1 and $6 \mathrm{~h}$ after NS or zymosan injection, respectively. At $24 \mathrm{~h}$ after NS or zymosan injection, all the animals were anesthetized, and the blood samples were collected for detection of the serum biochemical parameters.

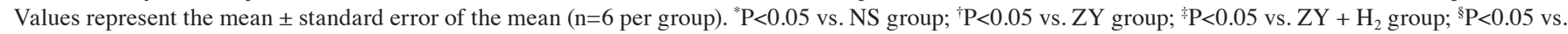
$\mathrm{ZY}+\mathrm{O}_{2}$ group. ALT, alanine aminotransferase; AST, aspartate aminotransferase; BUN, blood urea nitrogen; $\mathrm{Cr}$, creatinine; UI/L, international units per liter; NS, normal saline; ZY, zymosan.

with $98 \% \mathrm{O}_{2}$ and $2 \% \mathrm{H}_{2}$ (Fig. 3). These results demonstrate that combination therapy with $\mathrm{H}_{2}$ and hyperoxia has a more substantially beneficial effect on liver and kidney dysfunction in ZY-challenged mice.
Combination therapy with $\mathrm{H}_{2}$ and hyperoxia prevents the abnormal changes of antioxidant enzymatic activities, oxidative product and inflammatory cytokines in $Z Y$-challenged mice. The activity of the antioxidative enzyme SOD, the levels 
A

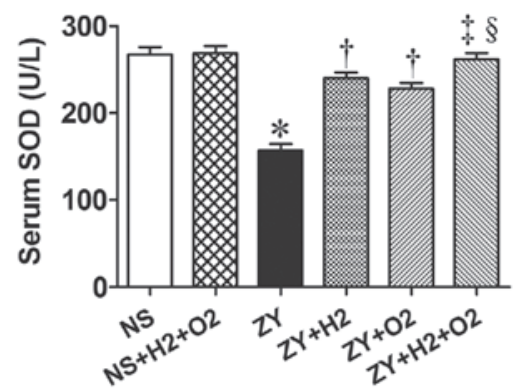

C

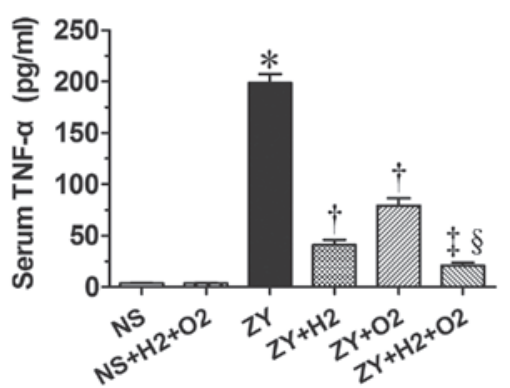

B

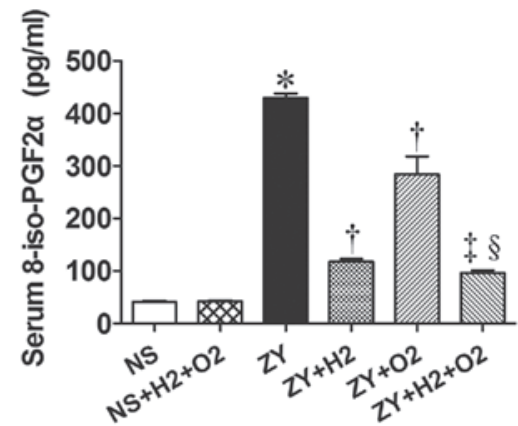

D

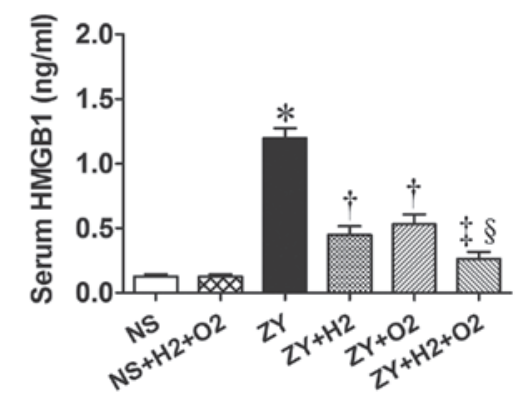

Figure 4. Effects of $\mathrm{H}_{2}$ and/or hyperoxia on the activity of serum antioxidant enzyme as well as the levels of serum oxidative product and inflammatory cytokines in zymosan-challenged mice. (A) SOD activity and serum levels of (B) 8-iso-PGF2 $\alpha$, (C) TNF- $\alpha$ and (D) HMGB1 level. The mice were treated with or without $2 \% \mathrm{H}_{2}$ and/or $98 \% \mathrm{O}_{2}$ inhalation for $3 \mathrm{~h}$ starting at 1 and $6 \mathrm{~h}$ after NS or zymosan injection, respectively. The serum was harvested for measuring these indicators at $24 \mathrm{~h}$ after NS or zymosan injection. Values are expressed as the mean \pm standard error of the mean (n=6 per group). ${ }^{*}<0.05$ vs. NS group; ${ }^{\dagger} \mathrm{P}<0.05$ vs. $\mathrm{ZY}$ group; ${ }^{\dagger} \mathrm{P}<0.05$ vs. $\mathrm{ZY}+\mathrm{H}_{2}$ group; ${ }^{\S} \mathrm{P}<0.05$ vs. $\mathrm{ZY}+\mathrm{O}_{2}$ group. SOD, superoxide dismutase; 8 -iso-PGF2 $\alpha, 8$-iso-prostaglandin F2 $\alpha$; HMGB1, high-mobility group box 1 protein; NS, normal saline; ZY, zymosan.

of the oxidative product 8 -iso-PGF $2 \alpha$ and the levels of proinflammatory cytokines (HMGB1 and TNF- $\alpha$ ) in serum of all animals were observed at $24 \mathrm{~h}$ after NS or ZY injection. The results showed decreased SOD activity and increased levels of 8 -iso-PGF2 $\alpha$, HMGB1 and TNF- $\alpha$ in the serum of the ZY-challenged mice ( $\mathrm{P}<0.05$ vs. NS group; Fig. 4). Furthermore, the results showed that $2 \% \mathrm{H}_{2}$ or $98 \% \mathrm{O}_{2}$ alone significantly increased the SOD activity and decreased the 8-iso-PGF $2 \alpha$ level in serum of $\mathrm{ZY}$-challenged mice $(\mathrm{P}<0.05$; Fig. 4). In addition, $2 \% \mathrm{H}_{2}$ or $98 \% \mathrm{O}_{2}$ alone significantly decreased the serum HMGB1 and TNF- $\alpha$ levels of the ZY-challenged mice $(\mathrm{P}<0.05$; Fig. 4). These abnormal changes in oxidative stress and inflammatory cytokines were more markedly improved by combination therapy with $98 \% \mathrm{O}_{2}$ and $2 \% \mathrm{H}_{2}$ (Fig. 4).

These data suggest that combination therapy with $\mathrm{H}_{2}$ and hyperoxia produces a more beneficial effect on survival rate and organ damage in ZY-challenged mice, which is associated with decreased levels of oxidative product and proinflammatory cytokines and increased levels of antioxidative enzymes in the serum.

\section{Discussion}

The present study demonstrated that either $\mathrm{H}_{2}$ or hyperoxia treatment alone improved the survival rate of $\mathrm{ZY}$-challenged mice, while combination therapy with $\mathrm{H}_{2}$ and hyperoxia could synergistically increase the survival rate of ZY-challenged mice, which was greater than treatment with either gas alone. Furthermore, $\mathrm{H}_{2}$ or hyperoxia treatment alone protected against lung, liver and kidney damage in ZY-challenged mice, while combination therapy with $\mathrm{H}_{2}$ and hyperoxia provided cumulative protection against these organ damage of ZY-challenged mice. In addition, the beneficial effects of combination therapy with $\mathrm{H}_{2}$ and hyperoxia on ZY-induced organ injury were associated with decreased levels of oxidative product 8 -iso-PGF2 $\alpha$, increased SOD activity and reduced levels of inflammatory cytokines TNF- $\alpha$ and HMGB1 in serum.

$\mathrm{ZY}$ is a substance derived from the cell wall of the yeast Saccharomyces cerevisiae, and can induce systemic inflammation by inducing a wide range of inflammatory mediators (18). Based on previous studies by the present authors and others, intraperitoneal injection of a high dose of ZY $(0.8-1.0 \mathrm{~g} / \mathrm{kg})$ can induce a generalized inflammation in rats or mice, which is accompanied by multiple organ damage $(8,17,18,22)$. ZY $(1.0 \mathrm{~g} / \mathrm{kg}$, intraperitoneal injection) successfully induced sterile inflammation model in mice, characterized by the decrease of survival rates, histopathological injury, organ dysfunction and abnormally decreased tissue oxygenation $(8,22)$. In the present study, similar changes were observed in the ZY-challenged mice.

Oxygen therapy is widely used in clinical practice as a mainstay of supportive treatment for patients with hypoxemia and critical illness. It is well known that early goal-directed therapy for MODS aims to balance oxygen delivery and demand $(24,25)$. Previous animal studies have shown that hyperoxia exposure can improve organ function and survival rate in several models of shock or sepsis $(4-8,26,27)$. In addition, $100 \%$ oxygen exposure for 2 and $3 \mathrm{~h}$ starting at 4 and $12 \mathrm{~h}$, respectively, after ZY injection benefits the outcome of mice with sterile sepsis (8). It is speculated that improved tissue oxygenation and decreased systemic inflammatory response are crucially involved in the protective effects of hyperoxia 
treatment (4-8). However, hyperoxia treatment can induce the production of ROS, which are considered to be associated with oxygen toxicity $(9,10)$. Therefore, the use of hyperoxia is limited in critically ill patients due to concerns that it may exacerbate organ damage by increasing free radical formation.

$\mathrm{H}_{2}$ has been used medically to prevent decompression sickness in deep divers for safety profiles (28). Prior studies have shown that $\mathrm{H}_{2}$ exerts a therapeutic antioxidative effect by selectively reducing ROS toxicity, and effectively protecting against a number of diseases, suggesting that $\mathrm{H}_{2}$ has potential as an antioxidant for therapeutic applications (11-15). Our previous studies have shown that $\mathrm{H}_{2}$ treatment has a beneficial effect on sepsis and sepsis-induced organ injury $(16,20,21)$. In the present study, combination therapy with $\mathrm{H}_{2}$ and hyperoxia appeared to produce a synergistic protective effect against sepsis and sepsis-associated multiple organ damage (16). The present results showed that combination therapy with $\mathrm{H}_{2}$ and hyperoxia had a more beneficial effect on multiple organ dysfunction/failure in the ZY-induced generalized inflammation model.

To further investigate the possible underlying mechanisms, the effects of $\mathrm{H}_{2}$ and/or hyperoxia treatment on oxidant and antioxidant system in ZY-challenged mice were investigated. In our previous studies, we found that the activities of SOD, CAT and GSH-Px in serum and tissues are significantly decreased during the early and late stages of ZY-induced organ damage, indicating that $\mathrm{ZY}$ sets up an environment favorable for oxidative stress $(8,22)$. In the present study, decreased SOD activity and increased 8 -iso-PGF2 $\alpha$ levels were detected in the serum at $24 \mathrm{~h}$ after $\mathrm{ZY}$ injection. Furthermore, $2 \% \mathrm{H}_{2}$ and/or $98 \% \mathrm{O}_{2}$ treatment significantly improved SOD activity and decreased the 8 -iso-PGF $2 \alpha$ levels in the serum. These results suggest that the reduction of oxidative damage and the increase of endogenous antioxidant enzymatic activities in the serum may associated with the protective effects of $\mathrm{H}_{2}$ and/or $\mathrm{O}_{2}$ treatment, which is consistent with our previous study (20). It has been hypothesized that the uncontrolled and exaggerated inflammatory response plays a major role in the pathogenesis of sepsis/MODS (3). Inflammatory cytokines include early inflammatory cytokines such as proinflammatory cytokines TNF- $\alpha$, IL-6 and anti-inflammatory cytokine IL-10, as well as the late inflammatory cytokine HMGB1 $(29,30)$. Early and late inflammatory cytokines can interact and facilitate the organ dysfunction and injury in sepsis/MODS. Our previous studies have demonstrated that HMGB1 contributed to organ damage in the ZY-induced generalized inflammation model $(8,22)$. In the present study, we found that ZY-challenged mice showed the significant increase of TNF- $\alpha$ and HMGB1 in serum, which was significantly attenuated by $2 \% \mathrm{H}_{2}$ and/or $\mathrm{O}_{2}$ treatment. These data suggest that the protective effects of combination therapy with $\mathrm{H}_{2}$ and hyperoxia treatment on ZY-challenged mice are associated with a reduction in the serum levels of early and late proinflammatory cytokines, which is consistent with our previous study $(20,21)$.

$\mathrm{H}_{2}$ is neither explosive nor dangerous at a low concentration $(<4.1 \%$ in oxygen). The present results suggest that a mixed gas therapy with $\mathrm{H}_{2}$ and hyperoxia may be a novel, safe and effective approach for preventing organ damage and mortality in MODS.

\section{Acknowledgements}

This study was supported by research grants from the National Natural Science Foundation of China (grant nos. 81101409 and 81471842) and Natural Science Foundation of Tianjin, China (no. 13JCQNJC11400), the Foundation of Tianjin Bureau of Public Health (no. 2011KZ108).

\section{References}

1. Martin GS, Mannino DM, Eaton S and Moss M: The epidemiology of sepsis in the United States from 1979 through 2000 N Engl J Med 348: 1546-1554, 2003.

2. Shayevitz JR, Miller C, Johnson KJ and Rodriguez JL: Multiple organ dysfunction syndrome: End organ and systemic inflammatory response in a mouse model of nonseptic origin. Shock 4: 389-396, 1995.

3. Hotchkiss RS and Karl IE: The pathophysiology and treatment of sepsis. N Engl J Med 348: 138-150, 2003.

4. Asfar P, Calzia E, Huber-Lang M, Ignatius A and Radermacher P. Hyperoxia during septic shock-Dr. Jekyll or Mr. Hyde? Shock 37: 122-123, 2012.

5. Waisman D, Brod V, Rahat MA, Amit-Cohen BC, Lahat N, Rimar D, Menn-Josephy H, David M, Lavon O, Cavari Y and Bitterman H: Dose-related effects of hyperoxia on the lung inflammatory response in septic rats. Shock 37: 35-102, 2012.

6. Hauser B, Barth E, Bassi G, Simon F, Gröger M, Oter S, Speit G, Ploner F, Möller P, Wachter U, et al: Hemodynamic, metabolic and organ function effects of pure oxygen ventilation during established fecal peritonitis-induced septic shock. Crit Care Med 37: 2465-2469, 2009.

7. Barth E, Bassi G, Maybauer DM, Simon F, Gröger M, Oter S, Speit G, Nguyen CD, Hasel C, Möller P, et al: Effects of ventilation with $100 \%$ oxygen during early hyperdynamic porcine fecal peritonitis. Crit Care Med 36:495-503, 2008.

8. Hou L, Xie K, Li N, Qin M, Lu Y, Ma S, Ji G and Xiong L: $100 \%$ oxygen inhalation protects against zymosan-induced sterile sepsis in mice: The roles of inflammatory cytokines and antioxidant enzymes. Shock 32: 451-461, 2009.

9. Folz RJ, Abushamaa AM and Suliman HB: Extracellular superoxide dismutase in the airways of transgenic mice reduces inflammation and attenuates lung toxicity following hyperoxia. J Clin Invest 103: 1055-1066, 1999.

10. Altemeier WA and Sinclair SE: Hyperoxia in the intensive care unit: Why more is not always better. Curr Opin Crit Care 13: 73-78, 2007.

11. Ohsawa I, Ishikawa M, Takahashi K, Watanabe M, Nishimaki K, Yamagata K, Katsura K, Katayama Y, Asoh S and Ohta S: Hydrogen acts as a therapeutic antioxidant by selectively reducing cytotoxic oxygen radicals. Nat Med 13: 688-694, 2007.

12. Ohta S: Hydrogen gas and hydrogen water act as a therapeutic and preventive antioxidant with a novel concept. Nippon Ronen Igakkai Zasshi 45: 355-362, 2008 (In Japanese).

13. Fukuda K, Asoh S, Ishikawa M, Yamamoto Y, Ohsawa I and Ohta S: Inhalation of hydrogen gas suppresses hepatic injury caused by ischemia/reperfusion through reducing oxidative stress. Biochem Biophys Res Commun 361: 670-674, 2007.

14. Cai J, Kang Z, Liu WW, Luo X, Qiang S, Zhang JH, Ohta S, Sun $\mathrm{X}, \mathrm{Xu} \mathrm{W}$, Tao $\mathrm{H}$ and Li R: Hydrogen therapy reduces apoptosis in neonatal hypoxia-ischemia rat model. Neurosci Lett 441: 167-172, 2008

15. Huang CS, Kawamura T, Toyoda Y and Nakao A: Recent advances in hydrogen research as a therapeutic medical gas. Free Radic Res 44: 971-982, 2010.

16. Xie K, Fu W, Xing W, Li A, Chen H, Han H, Yu Y and Wang G: Combination therapy with molecular hydrogen and hyperoxia in a murine model of polymicrobial sepsis. Shock 38: 656-663, 2012.

17. Cuzzocrea S, Costantino G, Mazzon E and Caputi AP: Protective effect of $\mathrm{N}$-acetylcysteine on multiple organ failure induced by zymosan in the rat. Crit Care Med 27: 1524-1532, 1999.

18. Volman TJ, Hendriks T and Goris RJ: Zymosan-induced generalized inflammation: Experimental studies into mechanisms leading to multiple organ dysfunction syndrome. Shock 23: 291-297, 2005. 
19. Humphreys K, Weingardt KR and Harris AH: Influence of subject eligibility criteria on compliance with National Institutes of Health guidelines for inclusion of women, minorities, and children in treatment research. Alcohol Clin Exp Res 31: 988-995, 2007.

20. Xie K, Yu Y, Pei Y, Hou L, Chen S, Xiong L and Wang G: Protective effects of hydrogen gas on murine polymicrobial sepsis via reducing oxidative stress and HMGB1 release. Shock 34: 90-97, 2010.

21. Xie K, Yu Y, Zhang Z, Liu W, Pei Y, Xiong L, Hou L and Wang G: Hydrogen gas improves survival rate and organ damage in zymosan-induced generalized inflammation model. Shock 34: 495-501, 2010

22. Hou L, Xie K, Qin M, Peng D, Ma S, Shang L, Li N, Li S, Ji G, $\mathrm{Lu} \mathrm{Y}$ and Xiong L: Effects of reactive oxygen species scavenger on the protective action of $100 \%$ oxygen treatment against sterile inflammation in mice. Shock 33: 646-654, 2010.

23. Dworski R, Roberts LJ II, Murray JJ, Morrow JD, Hartert TV and Sheller JR: Assessment of oxidant stress in allergic asthma by measurement of the major urinary metabolite of F2-isoprostane, 15-F2t-IsoP (8-iso-PGF2alpha). Clin Exp Allergy 31: 387-390, 2001.

24. Martin DS and Grocott MP: Oxygen therapy in critical illness: Precise control of arterial oxygenation and permissive hypoxemia. Crit Care Med 41: 423-432, 2013.
25. Rivers E, Nguyen B, Havstad S, Ressler J, Muzzin A, Knoblich B, Peterson E and Tomlanovich M; Early Goal-Directed Therapy Collaborative Group: Early goal-directed therapy in the treatment of severe sepsis and septic shock. N Engl J Med 345: 1368-1377, 2001.

26. Meier J, Kemming GI, Kisch-Wedel H, Blum J, Pape A and Habler OP: Hyperoxic ventilation reduces six-hour mortality after partial fluid resuscitation from hemorrhagic shock. Shock 22: 240-247, 2004

27. Sukhotnik I, Krausz MM, Brod V, Balan M, Turkieh A, Siplovich L and Bitterman H: Divergent effects of oxygen therapy in four models of uncontrolled hemorrhagic shock. Shock 18: 277-284, 2002

28. Kayar SR and Fahlman A: Decompression sickness risk reduced by native intestinal flora in pigs after $\mathrm{H} 2$ dives. Undersea Hyperb Med 28: 89-97, 2001.

29. Wang H, Bloom O, Zhang M, Vishnubhakat JM, Ombrellino M, Che J, Frazier A, Yang H, Ivanova S, Borovikova L, et al: HMG-1 as a late mediator of endotoxin lethality in mice. Science 285: 248-251, 1999.

30. Hou LC, Qin MZ, Zheng LN, Lu Y, Wang Q, Peng DR, Yu XP, Xin YC, Ji GL and Xiong LZ: Severity of sepsis correlated with the elevation of serum high-mobility group box 1 in rats. Chin Med J (Engl) 122:449-454, 2009. 